\title{
Study of the fractality in a magnetohydrodynamic shell model forced by solar wind fluctuations
}

\author{
Macarena Domínguez ${ }^{1}$, Giuseppina Nigro ${ }^{2}$, Víctor Muñoz $^{3}$, Vincenzo Carbone ${ }^{2}$, and Mario Riquelme ${ }^{1}$ \\ ${ }^{1}$ Departamento de Física, Facultad de Ciencias Físicas y Matemáticas, Universidad de Chile, 8370449 Santiago, Chile \\ ${ }^{2}$ Dipartimento di Fisica, Universita della Calabria, 87036 Rende CS, Italy \\ ${ }^{3}$ Departamento de Física, Facultad de Ciencias, Universidad de Chile, 7800003 Santiago, Chile
}

Correspondence: Macarena Domínguez (mdominguezv@ug.uchile.cl)

Received: 19 June 2019 - Discussion started: 18 September 2019

Revised: 26 December 2019 - Accepted: 21 January 2020 - Published: 8 April 2020

\begin{abstract}
The description of the relationship between interplanetary plasma and geomagnetic activity requires complex models. Drastically reducing the ambition of describing this detailed complex interaction and, if we are interested only in the fractality properties of the time series of its characteristic parameters, a magnetohydrodynamic (MHD) shell model forced using solar wind data might provide a possible novel approach. In this paper we study the relation between the activity of the magnetic energy dissipation rate obtained in one such model, which may describe geomagnetic activity, and the fractal dimension of the forcing.

In different shell model simulations, the forcing is provided by the solution of a Langevin equation where a white noise is implemented. This forcing, however, has been shown to be unsuitable for describing the solar wind action on the model. Thus, we propose to consider the fluctuations of the product between the velocity and the magnetic field solar wind data as the noise in the Langevin equation, the solution of which provides the forcing in the magnetic field equation.

We compare the fractal dimension of the magnetic energy dissipation rate obtained, of the magnetic forcing term, and of the fluctuations of $v \cdot b_{z}$, with the activity of the magnetic energy dissipation rate. We examine the dependence of these fractal dimensions on the solar cycle. We show that all measures of activity have a peak near solar maximum. Moreover, both the fractal dimension computed for the fluctuations of $v \cdot b_{z}$ time series and the fractal dimension of the magnetic forcing have a minimum near solar maximum. This suggests that the complexity of the noise term in the Langevin equation may have a strong effect on the activity of the magnetic energy dissipation rate.
\end{abstract}

\section{Introduction}

There are many investigations regarding the relation between interplanetary plasma parameters and the occurrence of geomagnetic events in the Earth's magnetosphere (Kane, 2005; Gonzalez et al., 1994, 2004; Tsurutani et al., 1988; Burton et al., 1975; Rathore et al., 2015; Snyder et al., 1963). Among these, Rathore et al. (2015) and Kane (2005) show a decrease in the antiparallel geomagnetic field, $B_{s}$, before the occurrence of the minimum of Dst. While Gonzalez et al. (1994, 2004) and Burton et al. (1975) introduce an energy balance equation where the Dst index and the rectified interplanetary electric field $\left(\mathrm{d} v \cdot B_{z}\right)$ are related.

The study of the fractal dimension in various fields has contributed to understanding diverse phenomena, adding a new, interdisciplinary perspective to nonlinear systems. For example, this approach has been used to study seismicity, to describe the distribution of epicenter and hypocenters in a given geographical zone (Pastén et al., 2011), or to consider the relationship between the fractal dimension of the spatial distributions of the aftershocks and the faults (Nanjo and Nagahama, 2004; Sahimi et al., 1993). It has also been used in the study of various catastrophic events such as seismic and epileptic shocks, where the fractality of the relevant time series has been analyzed to extract information on precursor activity (Eftaxias et al., 2006, 2008). In music, musical pieces have been characterized through fractal dimensions (Gündüz and Gündüz, 2005; Hsü and Hsü, 1990; Su and Wu, 2007). And in plasma physics, the use of fractal dimensions to understand plasma properties is becoming increasingly common (Chang, 1999; Macek et al., 2005; Szczepaniak and 
Macek, 2008; Chang and Wu, 2008; Neto et al., 2008; Materassi and Consolini, 2007; Zaginaylov et al., 2002; Carreras et al., 2000; Yankov, 1997; Dimitropoulou et al., 2009; McAteer et al., 2010; Domínguez et al., 2014, 2017, 2018).

Fractal dimensions can be calculated from either time series or spatial patterns. For instance, the fractal dimension of the time series of auroral electrojets (AEs), or from spatial data such as solar magnetograms, has shown interesting properties, being generally a non-integer value and less than the Euclidean dimension (Aschwanden and Aschwanden, 2008a, b; Kozelov, 2003; McAteer et al., 2005). Several studies have analyzed the relationship between the fractal and multifractal dimension with physical properties, which has provided a tool to predict events on the surface on the Sun (solar flares), the solar wind, and the Earth's magnetosphere (Dimitropoulou et al., 2009; Aschwanden and Aschwanden, 2008a; McAteer et al., 2010, 2005; Uritsky et al., 2006; Georgoulis, 2012; Conlon et al., 2008; Chapman et al., 2008; Kiyani et al., 2007).

There are many different methods to calculate the fractal and multifractal dimensions. In a previous work, we have studied the temporal evolution of solar and geomagnetic activity, by calculating a scatter-box-counting fractal dimension from solar magnetograms and Dst data (Domínguez et al., 2014). The fractal dimension of the Dst analysis decreases during magnetic storms, an effect that is consistently observed across several timescales, from individual storms to a complete solar cycle. Our results suggest that this definition of fractal dimension is an interesting proxy for complexity in the Sun-Earth system, not only for static data but also when the evolution of solar and geomagnetic activities are followed.

Moreover, in Domínguez et al. (2017), the authors show that the fractal dimension and the occurrence of the bursts in magnetic energy dissipation rate $\epsilon_{\mathrm{b}}(t)$ computed in a magnetohydrodynamic (MHD) shell model integration have correlations similar to those observed in geomagnetic and solar wind data. In that work, the forcing terms of the MHD shell model are provided by the solution of the Langevin equation, where a white noise is employed. That forcing, previously adopted, shows stationary statistical properties, hence revealing its inadequacy to describe the effect of solar wind on the magnetospheric activity. In order to mimic the evolution of the magnetospheric forcing due to the solar wind, in Domínguez et al. (2018) the MHD shell model has been forced using magnetic and velocity field data measured in the solar wind. This latter work shows a peak in the activity of $\epsilon_{\mathrm{b}}(t)$ near solar maximum, whereas the fractal dimension of the forcing magnetic field time series has a minimum near solar maximum.

Considering these results, in this paper we present an attempt to describe the complex interaction between solar wind and magnetosphere using a very simple model, where we employ $v \cdot b_{z}$ data to deduce a suitable forcing for the magnetic field evolution. In particular the fluctuations of $v \cdot b_{z}$ values inferred from solar wind data are introduced as the noise in the Langevin equation. Then, the solution of this latter equation provides a forcing that we introduce in the magnetic field equation. Thus, by using data that are related to the occurrence of geomagnetic activity, our aim is to investigate whether the statistical properties described in this model evolve because of the evolution in the statistical properties of the forcing term. In particular, in this paper we study whether there is a relationship between the fractality of the forcing, and the activity and the fractality of the dissipation.

The paper is organized as follows. In Sect. 2 we present the main features of the MHD shell model used to calculate the magnetic energy dissipation rate $\epsilon_{\mathrm{b}}(t)$, as well as the method used to modify the forcing term of the model. In Sect. 3 we describe the method to calculate the fractal dimension of the fluctuation of $v \cdot b_{z}$ used as a noise term in the Langevin equation. In Sect. 4 we describe the method to calculate the fractal dimension of the magnetic forcing term, and the energy dissipation rate obtained from the shell model. In Sect. 5 we present the definitions of the activity parameters used to analyze the energy dissipation rate. In Sect. 6, the results obtained are presented and finally, in Sect. 7, our conclusions are discussed.

\section{Shell model}

In general, shell models allow the nonlinear dynamics of fluid systems to be dealt with, reproducing relevant features of MHD turbulence even for high Reynolds numbers, which involve a large computational cost in direct numerical simulations (Boffetta et al., 1999). This is done by means of a set of equations - a simplified version of the Navier-Stokes system - which greatly reduces the available degrees of freedom (Obukhov, 1971; Gledzer, 1973; Yamada and Ohkitani, 1988).

In this work, we use the MHD Gledzer-Ohkitani-Yamada (GOY) shell model, which has been shown to be adequate to describe the dynamics of the energy cascade in MHD turbulence (Lepreti et al., 2004), dynamo effect (Nigro and Carbone, 2010; Nigro and Veltri, 2011), statistics of solar flares (Boffetta et al., 1999; Lepreti et al., 2004; Nigro et al., 2004), finite-time singularities in turbulent cascades (Nigro and Carbone, 2015) and to model the fractal features of a magnetized plasma (Domínguez et al., 2017, 2018).

This model is described in more detail in our previous works (Domínguez et al., 2017, 2018). Below we focus on the choice of forcing terms, which is relevant for the present study.

In the model, the wave-vector space ( $k$ space) is divided into $N$ discrete shells of radius $k_{n}=k_{0} 2^{n}(n=0,1, \ldots, N)$. Then, two complex dynamical variables $u_{n}(t)$ and $b_{n}(t)$, representing, respectively, velocity and magnetic field increments on an eddy scale $l \sim k_{n}^{-1}$, are assigned to each shell. 
The following set of ordinary differential equations describes the dynamical behavior of the model (Lepreti et al., 2004):

$$
\begin{aligned}
\frac{\mathrm{d} u_{n}}{\mathrm{~d} t} & =-v k_{n}^{2} u_{n}+i k_{n}\left\{\left(u_{n+1} u_{n+2}-b_{n+1} b_{n+2}\right)\right. \\
& -\frac{1}{4}\left(u_{n-1} u_{n+1}-b_{n-1} b_{n+1}\right) \\
& \left.-\frac{1}{8}\left(u_{n-2} u_{n-1}-b_{n-2} b_{n-1}\right)\right\}^{*}+f_{n}, \\
\frac{\mathrm{d} b_{n}}{\mathrm{~d} t} & =-\eta k_{n}^{2} b_{n}+i k_{n} \frac{1}{6}\left\{\left(u_{n+1} b_{n+2}-b_{n+1} u_{n+2}\right)\right. \\
& +\left(u_{n-1} b_{n+1}-b_{n-1} u_{n+1}\right) \\
& \left.+\left(u_{n-2} b_{n-1}-b_{n-2} u_{n-1}\right)\right\}^{*}+g_{n} .
\end{aligned}
$$

Here, $v$ and $\eta$ are, respectively, the kinematic viscosity and the resistivity; $f_{n}$ and $g_{n}$ are external forcing terms acting, respectively, on the velocity and magnetic fluctuations.

Initially, velocities in the second and fourth shell are set to complex random numbers, whereas the initial magnetic field fluctuations are set to zero, $b_{n}(t=0)=0$ (Domínguez et al., 2017, 2018).

Based on Domínguez et al. (2017), where a comprehensive analysis of the statistical properties of the shell model for various values of $v$ and $\eta$ is carried out, we set $v=\eta=10^{-4}$, as it is in the range where the model is able to best reproduce the intermittent behavior observed in magnetized plasmas. Given the values of the dissipative coefficients $v$ and $\eta$, we take $N=19$, also consistent with the choices in Domínguez et al. $(2017,2018)$, a value which guarantees a nonlinear range sufficiently large to describe the system dynamics. We then numerically integrate the shell model Eqs. (1)-(2), and we can calculate the magnetic energy dissipation rate defined as follows:

$\epsilon_{\mathrm{b}}(t)=\eta \sum_{n=1}^{N} k_{n}^{2}\left|b_{n}^{2}\right|$.

Note that a dissipation rate for the velocity field can also be defined. However, this is not relevant to our model, as it would be related to heating, whereas there is no equation for temperature in our analysis. Magnetic storms, on the other hand, are related to magnetic dissipation rates.

In previous work (Domínguez et al., 2017; Lepreti et al., 2004; Nigro et al., 2004; Nigro and Carbone, 2010, 2015; Nigro and Veltri, 2011; Nigro, 2013) the forcing terms were obtained from the Langevin equation

$$
\frac{\mathrm{d} \tilde{f}_{n}}{\mathrm{~d} t}=-\frac{\tilde{f}_{n}}{\tau_{0}}+\mu(t)
$$

where $\tilde{f}_{n}=f_{n}$ or $g_{n}, \tau_{0}$ is a correlation time introduced in a Gaussian white noise $\mu$ of width $\sigma$. This provides a stochastic way to drive turbulence in the model. However, turbulence in space plasmas is not always subject to stationary drivers. Such is the case, for instance, of the Earth's magnetosphere. This system is driven by the solar wind, which itself has its own dynamics on short timescales due to local events such as coronal mass ejections (CMEs), and on longer timescales as the solar cycle. In this paper we deal with this property of the drivers, by considering a non-stationary forcing of the shell model.

One possible way of characterizing the stationarity of the forcing given by Eq. (4) is by calculating its fractal dimension, which is a simple measure of the complexity of the time series. Following Domínguez et al. (2014, 2017), a scatter plot is built from the time series, and then the box-counting fractal dimension of this plot is calculated and associated with the time series. When this method is applied to the output of Eq. (4) in various time windows, a value of $D \sim 1.7$ is obtained. Its independence of the used time window is a manifestation of the stationary character of the time series.

A first method to change the fractality of the forcing terms was presented in Domínguez et al. (2018). In that case, from two scalars - the flow speed and the average magnetic field of the solar wind as obtained by OMNI (https://cdaweb.gsfc. nasa.gov/istp_public/, last access: 4 March 2020), two complex series $f_{1}$ and $g_{1}$ were built, which were used as forcing of the first shell in Eqs. (1)-(2). In this way, it was shown that the activity of the resulting $\epsilon_{\mathrm{b}}(t)$ time series has a peak near solar maximum. However, the magnetic field time series seems to be the most sensitive, because its fractal dimension seems to correlate with the solar cycle much more than the fractal dimension of the velocity field time series. In fact, the latter does not show any particular sensitivity to the solar magnetic activity.

We now explore a second possibility to change the fractality of the forcing terms, namely, changing the method to calculate the stochastic term for magnetic forcing, which corresponds to $\mu(t)$ in Eq. (4). The conventional method to solve this equation, as mentioned above, considers $\mu(t)$ as a white Gaussian noise (Nigro et al., 2004; Lepreti et al., 2004).

Usually the forcing is applied only on the velocity equation, while in Domínguez et al. (2017) this forcing is considered for both the velocity and magnetic field equation.

Here, we will preserve the Gaussian noise for the velocity field, while for the magnetic field forcing we will use the fluctuations in $v \cdot b_{z}$,

$\mu(t)=v \cdot b_{z}-\left\langle v \cdot b_{z}\right\rangle$,

where $v$ and $b_{z}$ are the velocity and $z$-component magnetic field of the solar wind, respectively. This difference between the velocity and magnetic field forcing is because, as mentioned before, the velocity time series does not show any relation with the solar magnetic activity (Domínguez et al., 2018).

The data of the solar wind used in this work are obtained from the OMNIWeb Plus data service (https://cdaweb.gsfc. 
nasa.gov/istp_public/, last access: 4 March 2020). We consider this source because OMNI is a compilation of data obtained from many space missions (IMP 8, Geotail, Wind and ACE) of the magnetic field of the solar wind near the Earth. More specifically, we use data of $v$ and $b_{z}$ at 1 AU of distance with $1 \mathrm{~min}$ of resolution. The coordinate system of the data is the geocentric solar ecliptic (GSE). Thus, the $z$ axis is the projection of the axis of the Earth's magnetic dipole (positive to the north) on the plane perpendicular to the $x$ axis (towards the Sun).

Given that the forcing term must be a complex number in Eq. (2), a random phase $\varphi$ is needed for each datum that is calculated from Eq. (4) for the magnetic case. Then,

$$
f_{\mathrm{b}}(t)=\tilde{f}_{\mathrm{b}}(t) e^{2 \pi i \varphi}
$$

where the amplitude $\tilde{f}_{\mathrm{b}}(t)$ corresponds to the solution of the Langevin equation using the modified $\mu(t)$, and $f_{\mathrm{b}}(t)$ is the force term used in the shell model code.

In order to account for the variability with solar activity, we generate 13 time series of the magnetic energy dissipation, each one using the data corresponding to the 13 years of the 23rd solar cycle (1996 to 2008). Once the time series are generated, we define four indices to measure the activity of the magnetic energy rate $\left(\epsilon_{\mathrm{b}}\right)$ and analyze the relationship between these and the fractality of the data.

\section{Box-counting dimension of the fluctuation of $\boldsymbol{v} \cdot \boldsymbol{b}_{z}$}

In this work, we use the same definition as in Domínguez et al. $(2014,2017)$ for the scatter plot box-counting fractal dimension. The fractal dimension for each time series of the fluctuation of solar wind data (see Eq. 5) is estimated from their scatter diagram. If $\bar{\mu}^{i}$ is the $i$ th $\bar{\mu}$ datum in the series, and $\bar{N}$ is the total number of data, the scatter diagram is a plot of $\bar{\mu}^{1+(i+1) j}$ versus $\bar{\mu}^{1+i \cdot j}$, for $0 \leq i \leq(\bar{N}-1) / j$ and with a $j$ integer.

Then, the scatter diagram is divided in square cells of a certain size $\varepsilon$, and we count the number $\bar{N}(\varepsilon)$ of cells which contain a point. Next, we consider several values of $\varepsilon$, and we find the range of $\varepsilon$ where $\log (\bar{N}(\varepsilon))$ scales linearly with $\log \varepsilon$. If the slope in this region is given by $-D_{j}$, then in this region,

$\bar{N}(\epsilon) \propto \varepsilon^{-D_{j}}$.

Figure 1 illustrates the three steps to calculate the fractal dimension, using data for year 2000. Two values of $j$ are used as an example, $j=1$ and $j=10$.

\section{Box-counting dimension of the magnetic forcing term and the energy dissipation rate}

We used the same definition as in the previous section to calculate the fractal dimension of $\tilde{f}_{\mathrm{b}}(t)$ and $\epsilon_{\mathrm{b}}(t)$. Using 1 year of data of $v \cdot b_{z}$ fluctuations as the magnetic field forcing term, we obtain a $\tilde{\tilde{f}}_{\mathrm{b}}(t)$, and an energy dissipation rate time series. Then, for a given data window in this series, we construct the scatter plot and calculate its box-counting fractal dimension as described in Sect. 3.

Figure 2 illustrates these three steps to calculate the fractal dimension of the $\epsilon_{\mathrm{b}}$ time series. We can see that due to the very high time resolution in our computer simulation, necessary to properly solve the shell model equations, the change in $\epsilon_{t}(t)$ at each iteration is very small. This leads to a scatter plot for $j=1$, which is essentially a straight line of slope 1 , and thus to a box-counting dimension equal to 1 as well. However, for larger values of $j$ the scatter diagram presents a nontrivial structure.

\section{Activity parameters}

Some studies have reported a variation in the (multi)fractal features of the solar wind within the solar cycle (Szczepaniak and Macek, 2008; Macek, 2006, 2007; Macek and Wawrzaszek, 2009). On the other hand, it is well established that geomagnetic activity increases during solar maximum, and various models attempt to correlate specific features of the solar wind with geomagnetic activity (Gonzalez et al., 2004; Rathore et al., 2014; Kane, 2005; Huttunen et al., 2002; Rangarajan and Barreto, 2000; Echer et al., 2004). In this section we investigate whether the amount of complexity in the shell model forcing (as measured by its fractal dimension) somehow correlates with the level and complexity of the dissipation activity.

To this end, we need to define activity parameters for the output time series. We use the same parameters as in Domínguez et al. (2018). First, a threshold $\tilde{\epsilon}$ is chosen, so that an "active state" is said to appear whenever $\epsilon_{\mathrm{b}}(t)>\tilde{\epsilon}$. Then, four activity parameters are defined:

- $N$ is the number of data above that threshold.

- $\left\langle\epsilon_{\mathrm{b}}\right\rangle$ is the average of the data.

- $\left\langle\epsilon_{\mathrm{b}}\right\rangle_{\text {up }}$ is the average of the data above the threshold.

- $\max \left(\epsilon_{\mathrm{b}}\right)$ is the maximum value of $\epsilon_{\mathrm{b}}$.

The threshold was defined as follows:

$\tilde{\epsilon}_{\mathrm{b}}=\left\langle\epsilon_{\mathrm{b}}\right\rangle+n \cdot \sigma$,

where $\sigma$ is the standard deviation of the time series and $n$ is a number between 1 and 10. (In Domínguez et al., 2017 only $n=5$ and $n=10$ were considered.)

\section{Results}

We first study the fractal dimension of the noise term used to solve the magnetic Langevin equation, namely $\mu(t)$. In 

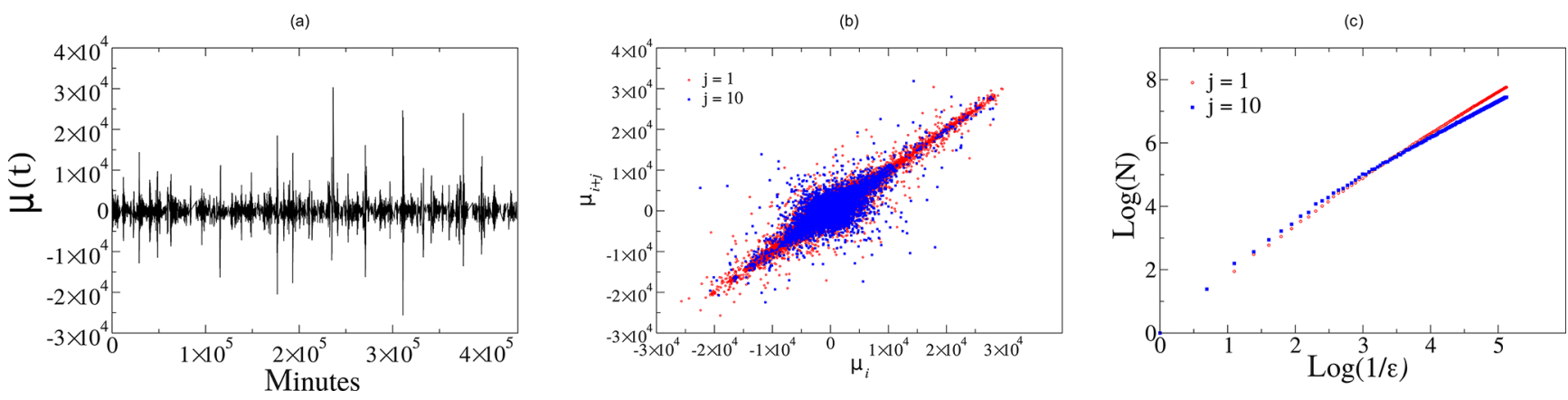

Figure 1. (a) $v \cdot b_{z}-\left\langle v \cdot b_{z}\right\rangle$ for the year 2000; (b) scatter diagram; (c) log-log plot of Eq. (7). Results for two values of the data sampling are shown: $j=1$ (red points) and $j=10$ (blue points).

(a)

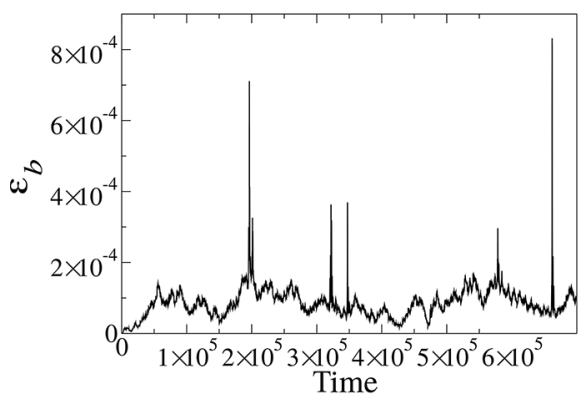

(b)

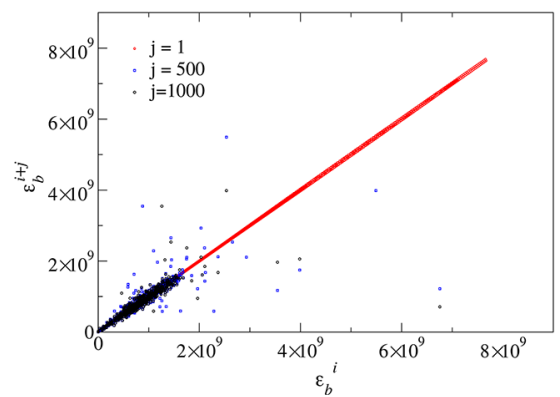

(c)

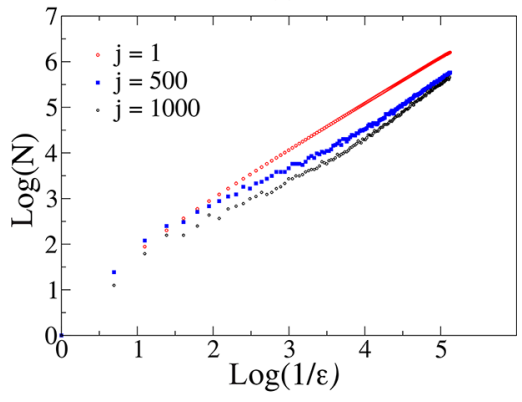

Figure 2. (a) $\epsilon_{\mathrm{b}}$ for the year 2000; (b) scatter diagram; (c) $\log -\log$ plot of Eq. (7). Results for three values of the data sampling are shown: $j=1$ (red points), $j=500$ (blue points), and $j=1000$ (black points).

general, the fractal dimension of the time series is expected to depend on the value of the time delay $j$. Thus we study its dependence on $j$, as well as its dependence on the solar cycle. Results are shown in Fig. 3.

Figure 3a shows that there is a general trend for the fractal dimension to decrease with $j$. Previous results based on the shell model simulation (Domínguez et al., 2017) show that active and quiet states can be distinguished by their different behavior with $j$ (decreasing or increasing its fractal dimension for intermediate values of $j$, respectively). However, all curves in Fig. 3a have the same trend, so it would seem that the fractal properties of the time series do not depend on the stage of the solar cycle.

However, after adding the information on sunspot activity, results are more clear. In order to show that, we take sunspot number data obtained from National Geophysical Data Center, prepared by the US Department of Commerce, NOAA, Space Weather Prediction Center (SWPC) (ftp://ftp.swpc. noaa.gov/pub/weekly/RecentIndices.txt, last access: 4 March 2020). By inspection, we note that the yearly average number of sunspots near solar minimum is below 40, whereas it quickly increases above 50 when approaching solar maximum, reaching 178 in 2002. Thus we set $N_{\mathrm{s}}=40$ as the threshold. If the number of sunspots in a year is greater than $N_{\mathrm{s}}$, the year is classified as closer to solar maximum; if the number of sunspots is less than $N_{\mathrm{s}}$, it is classified as closer to solar minimum. With this criterion, the years 1996, 1997, and 2006-2008 are classified in the minimum and the years 1998-2005 in the maximum of the solar cycle. The result is shown in Fig. 3b, where we clearly see that, on average, the fractal dimension of the fluctuations $\mu(t)$ discriminates between solar cycle minimum and maximum curves. The Fig. $3 \mathrm{~b}$ also shows that, when $j$ increases, the distinction between the minimum and maximum years improves. Then, henceforward we use $j=100$ to illustrate our findings.

We intend to compare the degree of complexity of the input time series with the level of activity in the dissipated magnetic energy. We have proposed four ways to measure activity in Sect. 5. For each year of the 23rd solar cycle, the solar wind fluctuation time series $\mu(t)$ is used to force the shell model, and the resulting activity in the output is measured.

As stated in Sect. 5, there are two parameters of the activity that depend of the threshold $\tilde{\epsilon}_{\mathrm{b}}$. With the aim of selecting the appropriate value of $n$ in Eq. (8), in Fig. 4 we first show the results for two of the activity parameters $N$ and $\left\langle\epsilon_{\mathrm{b}}\right\rangle_{\text {up }}$. We note that for $n=5,\left\langle\epsilon_{\mathrm{b}}\right\rangle_{\text {up }}$ has a clear peak near the solar maximum (year 2002, Fig. 4c). If the threshold is too large (Fig. 4d), sometimes no data are found above it, and the activity parameter drops to zero. In the case of $N$ (Fig. 4a, b), the curves for all values of $n$ yield similar results. No curve has a clear maximum near solar maximum, suggesting that 
(a)

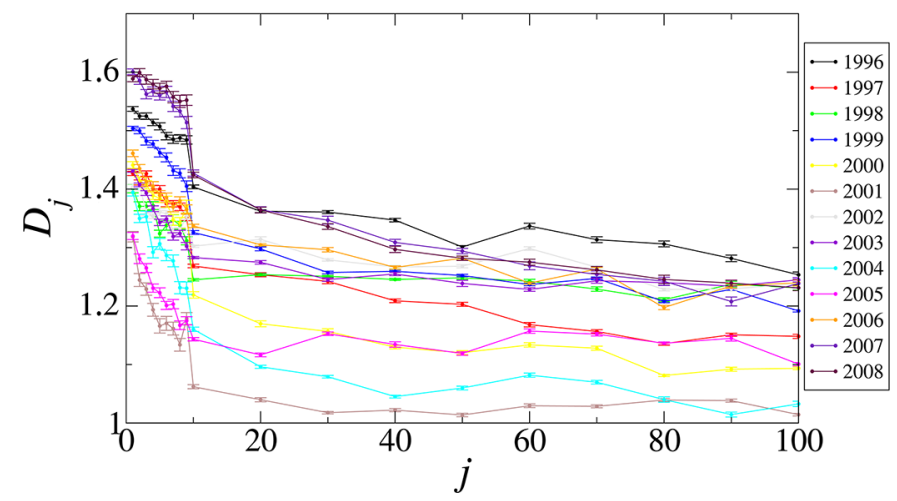

(b)

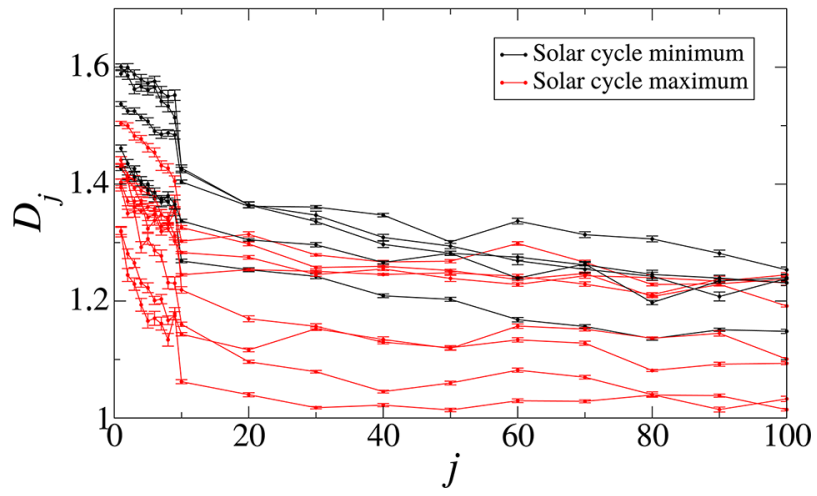

Figure 3. Box-counting dimension of $\mu(t)$ for different values of $j$. (a) Curves for each year of the 23rd solar cycle. (b) Curves are distinguished for years corresponding to the maximum (black lines: years 1998 to 2005) or minimum (red lines: years 1996, 1997, and 2006 to 2008) of the solar cycle.
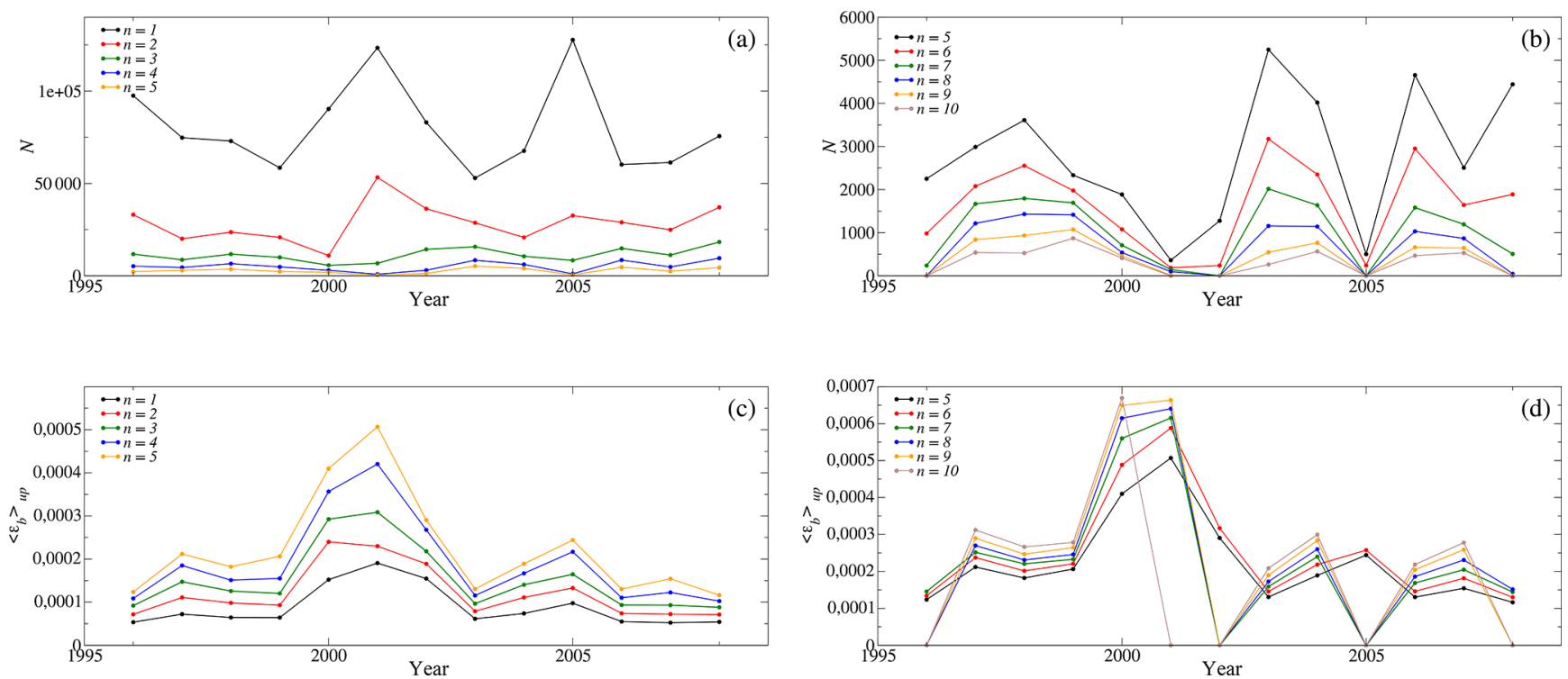

Figure 4. $N$ and $\left\langle\epsilon_{\mathrm{b}}\right\rangle_{\text {up }}$ calculated from the 13 time series of $\mu(t)$ for different values of $n$. For clarity, separate plots are shown for two sets of values for $n$ : from 0 to $5(\mathbf{a}, \mathbf{c})$, and from 5 to $10(\mathbf{b}, \mathbf{d})$. The grey region corresponds to years of maximum solar activity, as described in the text.

this parameter is rather insensitive to solar activity. Note that in Domínguez et al. (2018), $N$ was the parameter that showed the strongest correlation with the solar cycle. This highlights the complexity in the definition of a suitable metric for activity. On the other hand, Fig. 4 shows that the model does respond to various activity levels in the forcing time series, regardless of whether such forcing involves the fields themselves (Domínguez et al., 2018) or their fluctuations (this work).

Based on the previous discussions, we conclude that a moderate value of $n$ is appropriate when defining the activity parameters, so that the anomalous behavior in Fig. $4 b, d$ is avoided. We will take $n=5$.
We now compare the various activity parameters with the fractal dimension of $\mu(t)$. Figures 5 and 6 show the fractal dimension of $\mu(t)$, and one of the activity parameters for each time series. We see that, in general (except for $N$ ), the maximum of the activity parameters computed for $\epsilon_{\mathrm{b}}(t)$ approximately occurs in the years around the solar maximum. Moreover, the fractal dimension of $\mu(t)$ decreases during the same period.

We perform a similar analysis, but for the fractal dimension of the magnetic forcing term, $\tilde{f}_{\mathrm{b}}(t)$ in Eq. (6). That is, we calculate the dependence of the fractal dimension on $j$ for the $\tilde{f}_{\mathrm{b}}(t)$ time series and relate it to the stage within the solar cycle as was done in Fig. 3b. Results are shown in Fig. 7. 

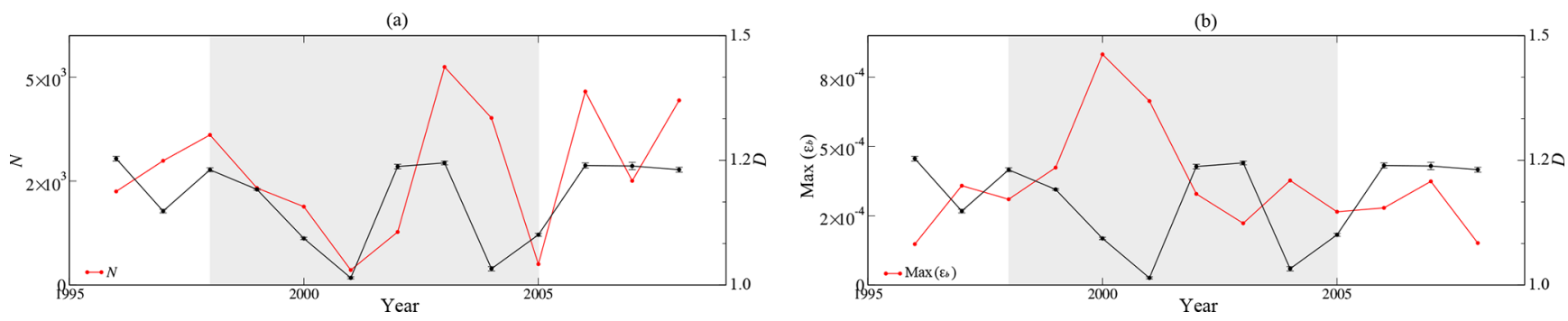

Figure 5. Box-counting dimension of $\mu(t)$ for the solar wind with $j=100$ (black line), and the activity of the dissipated magnetic energy $\epsilon_{\mathrm{b}}(t)$ (red line), as measured by the parameter $N$ (a) and $\max \left(\epsilon_{\mathrm{b}}\right)(\mathbf{b})$. Threshold to define activity is $n=5$ (see Eq. 8). The grey region corresponds to years of maximum solar activity, as described in the text.
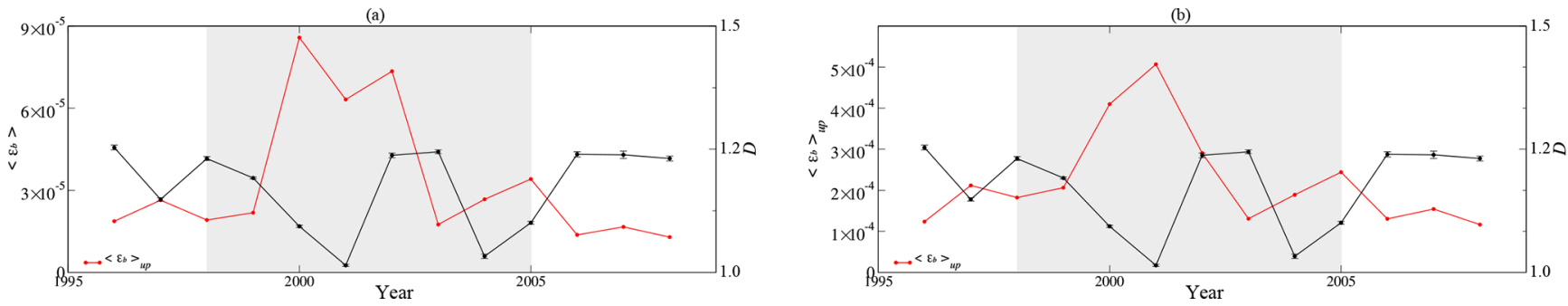

Figure 6. Same as Fig. 5, but for activity parameters $\left\langle\epsilon_{\mathrm{b}}(t)\right\rangle$ (a) and $\left\langle\epsilon_{\mathrm{b}}\right\rangle_{\mathrm{up}}$ (b). The grey region corresponds to years of maximum solar activity, as described in the text.

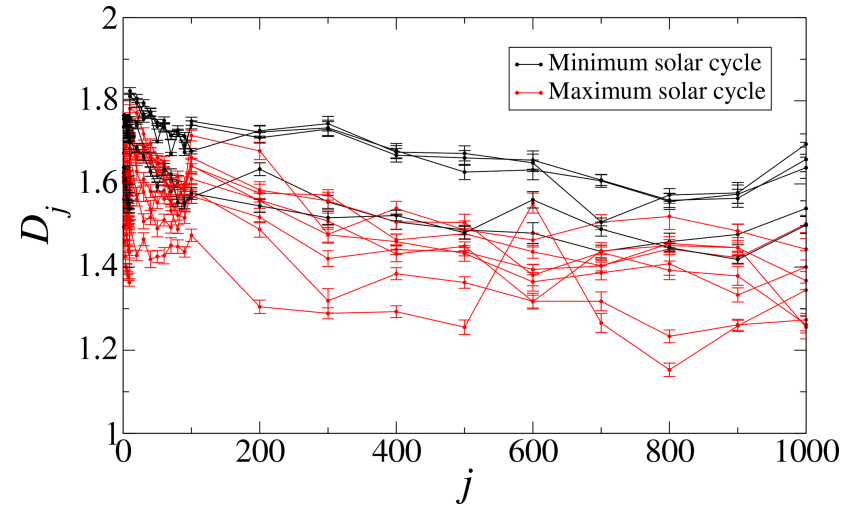

Figure 7. Box-counting dimension of magnetic forcing term $\tilde{f}_{\mathrm{b}}(t)$ for different values of $j$. Curves are distinguished for years corresponding to maximum (black lines: years 1998 to 2005) or minimum (red lines: years 1996, 1997, and 2006 to 2008) of the solar cycle.

The conclusion from Fig. 7 is similar to the one deduced in the previous analysis for $\mu(t)$ (Fig. 3). The general trend of the fractal dimension is to decrease with $j$, and the fractal dimension of the magnetic forcing during years close to solar minimum is, in general, larger than the one measured during the years near solar maximum. In Fig. 7, this is more clear for $j>300$.

Considering the above results, we now choose $j=500$ for the following figures. In Figs. 8 and 9, we compare the fractal dimension of the magnetic forcing term $\tilde{f}_{\mathrm{b}}(t)$ with $j=500$ for each year, with the same activity parameters of Figs. 5 and 6. We can see that, like the previous analysis, the fractal dimension of the magnetic forcing term has a minimum in the years of maximum activity. Also, consistent with Figs. 5 and 6, results for $N$ are less clear, as seen in Fig. 8.

Finally, we perform the analysis of the fractal dimension of the magnetic energy dissipation rate $\epsilon_{\mathrm{b}}(t)$ for different values of $j$. This latter fractal dimension, depicted in Fig. 10, does not show any particular dependence on the solar cycle, at least during the 23 rd solar cycle here considered.

It is important to note that for small values of $j$, the fractal dimension is essentially constant. In fact, for $j=1$ the fractal dimension is always one for all years, due to the scatter diagram being exactly a line (see Fig. 2). Unlike Fig. 7, Fig. 10 does not suggest a robust correlation between the fractal dimension of $\epsilon_{\mathrm{b}}(t)$ and the solar cycle, for any value of $j$.

Therefore, the time-dependent fractal dimension that characterized the forcing adopted here leads to noticeable variations in the intermittency of the magnetic energy dissipation rate, as measured by the activity parameters defined above. On the other hand, the same quantity, namely magnetic energy dissipation rate, does not show any significant variations of its fractal dimension during the cycle considered.

It is interesting to discuss Figs. 3, 7, and 10 in the light of comparative studies of complexity in the solar wind and the magnetosphere, although this work attempts to make a very simplified model of the interaction between the solar wind and the Earth's magnetosphere. In effect, Figs. 3 and 7 show the complexity of the drivers of the shell model, which we 

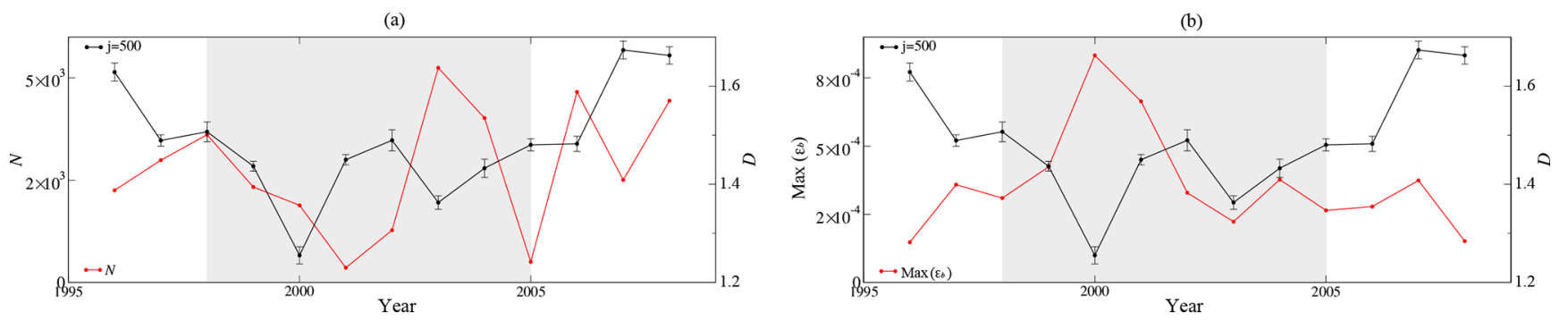

Figure 8. Box-counting dimension of magnetic forcing term for $j=500$ with respective activity of $\epsilon_{\mathrm{b}}(t)\left(\right.$ red lines): $N$ (a) and max $\left(\epsilon_{\mathrm{b}}\right)(\mathbf{b})$, with $n=5$. The grey region corresponds to the maximum period of the solar cycle, as described in the text.
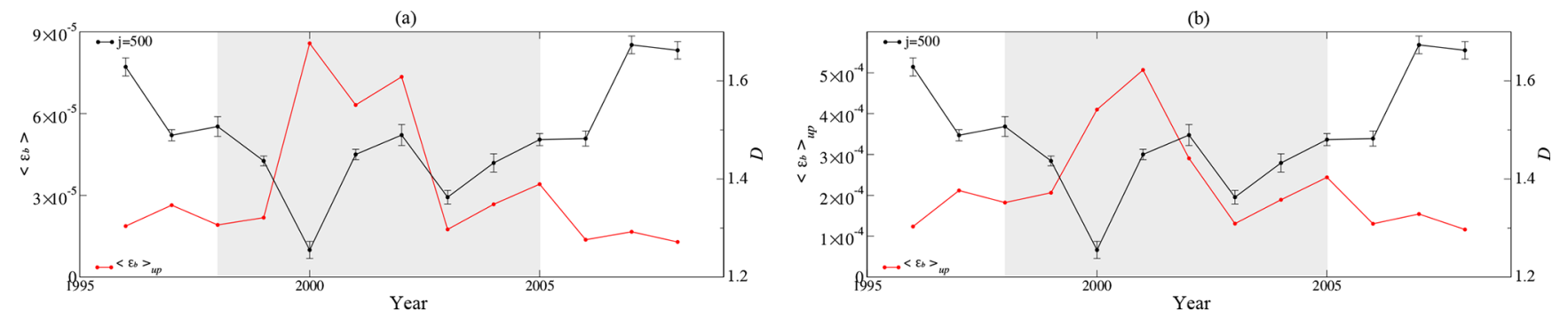

Figure 9. Box-counting dimension of magnetic forcing term for $j=500$ with respective activity of $\epsilon_{\mathrm{b}}(t)$ (red lines): $\left\langle\epsilon_{\mathrm{b}}(t)\right\rangle(\mathbf{a})$ and $\left\langle\epsilon_{\mathrm{b}}\right\rangle_{\text {up }}(\mathbf{b})$, with $n=5$. The grey region corresponds to the maximum period of the solar cycle, as described in the text.

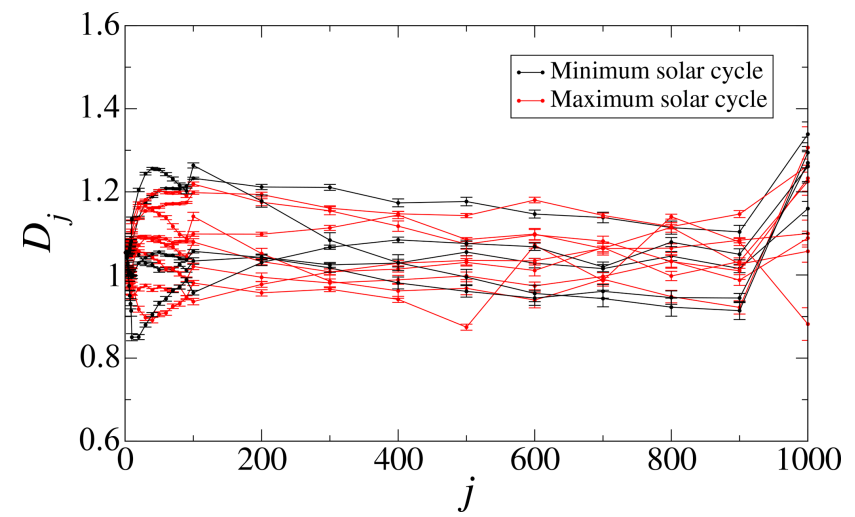

Figure 10. Box-counting dimension of energy dissipation rate for different values of $j$. Curves are distinguished for years corresponding to maximum (black lines: years 1998 to 2005) or minimum (red lines: years 1996, 1997, and 2006 to 2008) of the solar cycle.

may loosely associate with the solar wind driving the magnetosphere, whereas Fig. 10 shows the complexity of the output of the shell model, which may represent the magnetospheric activity, following the analogy.

Thus, these plots show that the complexity of the driven system (Fig. 10) is more similar to the complexity of the driver (Figs. 3, 7) during solar maximum than during solar minimum, and that the complexity of the driven system is typically lower than the complexity of the driver. This is different from results in Balasis et al. (2006), where a study in terms of Hurst exponents was made, finding that complexity in the magnetosphere is larger than in the solar wind. However, it should also be noted that in our work, longer-term trends are studied (1-year windows), instead of timescales of the order of the duration of geomagnetic storms. The different timescales, and the use of different metrics for complexity, could be relevant to compare both results.

\section{Conclusions}

In this paper we present the results of an MHD shell model where we force the velocity field fluctuations and the magnetic field fluctuations differently. In particular, while the forcing employed in the velocity equation is a timecorrelated Gaussian noise, for the magnetic field equation we adopt the solution of a Langevin equation where the fluctuations of $v \cdot b_{z}$, computed using solar wind data, are introduced in this equation instead of a stochastic term. This produces a forcing on the magnetic field equation that mimics the timedependent solar wind action on Earth's magnetosphere during a solar cycle. This description is certainly an oversimplification of the complex dynamics that determine the interaction between solar wind and Earth's magnetosphere, but it provides a possible approach if we are interested only in the fractal properties of the time series of the characteristic parameters.

In this framework, we have analyzed the relationships of the activity of the magnetic energy dissipation rate obtained in the shell model, with the fractal dimension of its input and output time series. Specifically, our defined activity parame- 
ters are compared with the fractal dimension of the fluctuations of the solar wind $v \cdot b_{z}$ data, the magnetic force term, and the time series of the magnetic energy dissipation rate.

Both the fluctuation term, $\mu(t)$, and the resulting forcing term (Eqs. 5 and 6) have a fractal dimension which is well correlated with the solar cycle, as shown in Figs. 3 and 7, indicating that information on solar activity is actually present in the fractal dimension of $\mu(t)$ and resulting forcing. This is not the case for the magnetic energy dissipation rate, as can be seen in Fig. 10. Thus, this complexity measure produces signatures of the corresponding solar activity when applied to the input of the shell model, but does not produce them when applied to the output of the model.

For the quantities which possess a time-dependent fractal dimension, namely $\mu(t)$ and the forcing term, this dimension exhibits a minimum near solar maximum. As to the activity of the output, all proposed metrics - except $N$ - seem to correlate with the solar cycle, showing a peak near the solar maximum. This suggests that the complexity of the noise term of Langevin equation may have, within the simulation, a noticeable effect in the activity of the magnetic energy dissipation rate, although the fractal dimension, as calculated here, is not a suitable metric for that output activity.

Despite this, it is interesting to see that some results are consistent with previous studies, based directly on data. Fractal dimensions in Figs. 3 and 7 measure the complexity of the drivers of the shell model, which we may loosely associate with the solar wind driving the magnetosphere, whereas Fig. 10 measures the complexity of the output of the shell model, which may represent the magnetospheric activity, following the analogy. Results are similar to those calculated for the solar wind (Domínguez et al., 2018) and for the Dst index (Domínguez et al., 2014), in the sense that values of the fractal dimensions suggest that the complexity of the solar wind is larger than the complexity of the magnetosphere, measured using the same box-counting approach as presented here.

Given the complex dynamics in the system studied, we should not expect that a single metric contains all the information, and thus results may depend on the method used. For instance, Hurst exponents are used in Balasis et al. (2006), which suggests that complexity in the magnetosphere is larger than in the solar wind. On the other hand, the timescales observed are also different from those in Balasis et al. (2006), and this can also be relevant to evaluate complexity in a physical system.

Nevertheless, it is interesting that various studies have considered the use of fractal dimensions, using several strategies, as a means to extract information on solar windmagnetosphere interaction, either in the sense of precursor activity (Donner et al., 2018; Balasis et al., 2006), or longerterm trends (Domínguez et al., 2014, 2018). Simulationbased studies may help to understand to what extent complexity measures may be relevant for this task.
Data availability. OMNI data for the flow speed and average magnetic field of the solar wind can be downloaded from the website of the Coordinated Data Analysis Web (CDAWeb), Goddard Space Flight Center, https://cdaweb.gsfc.nasa.gov/istp_public/ (last access: 4 March 2020, OMNIWeb Plus Data Service, 2020). Sunspot number data can be downloaded from the website of the Space Weather Prediction Center (SWPC, 2020), ftp://ftp.swpc.noaa.gov/ pub/weekly/RecentIndices.txt (last access: 4 March 2020).

Author contributions. MD did the main numerical analysis and was involved in editing the paper and in scientific discussions for the whole text. VM was involved in writing and editing the paper and in scientific discussions for the whole text. GN and VC were involved in the creation of the numerical code, in editing the paper, and in scientific discussions for the whole text. MR was involved in editing the paper, and in scientific discussions for the whole text.

Competing interests. The authors declare that they have no conflict of interest.

Acknowledgements. We thank the support of CONICYT through FONDECYT grant nos. 1161711 and 1201967 (Víctor Muñoz), grant no. 3160305 (Macarena Domínguez), and grant no. 1191673 (Mario Riquelme).

Financial support. This research has been supported by the Fondo Nacional de Desarrollo Científico y Tecnológico, FONDECYT (grant nos. 1161711, 1201967, 3160305, and 1191673).

Review statement. This paper was edited by Bruce Tsurutani and reviewed by two anonymous referees.

\section{References}

Aschwanden, M. J. and Aschwanden, P. D.: Solar Flare Geometries. I. The Area Fractal Dimension, Astrophys. J., 674, 530 543, https://doi.org/10.1086/524371, 2008a.

Aschwanden, M. J. and Aschwanden, P. D.: Solar Flare Geometries. II. The Volume Fractal Dimension, Astrophys. J., 674, 544-553, https://doi.org/10.1086/524370, 2008b.

Balasis, G., Daglis, I. A., Kapiris, P., Mandea, M., Vassiliadis, D., and Eftaxias, K.: From pre-storm activity to magnetic storms: a transition described in terms of fractal dynamics, Ann. Geophys., 24, 3557-3567, https://doi.org/10.5194/angeo-24-35572006, 2006.

Boffetta, G., Carbone, V., Giuliani, P., Veltri, P., and Vulpiani, A.: Power Laws in Solar Flares: Self-Organized Criticality or Turbulence?, Phys. Rev. Lett., 83, 4662-4665, https://doi.org/10.1103/PhysRevLett.83.4662, 1999.

Burton, R. K., McPherron, R. L., and Russel, C. T.: An Empirical Relationship between Interplanetary Con- 
ditions and Dst, J. Geophys. Res., 80, 4204-4217, https://doi.org/10.1029/JA080i031p04204, 1975.

Carreras, B. A., Lynch, V. E., Newman, D. E., Balbín, R., Bleuel, J., Pedrosa, M. A., Endler, M., van Milligen, B., Sánchez, E., and Hidalgo, C.: Intermittency of Plasma Edge Fluctuation data: Multifractal Analysis, Phys. Plasmas, 7, 3278-3287, https://doi.org/10.1063/1.874193, 2000.

Chang, T.: Self-Organized Criticality, Multi-Fractal Spectra, Sporadic Localized Reconnection and Intermittent Turbulence in the Magnetotail, Phys. Plasmas, 6, 4137, https://doi.org/10.1023/A:1002486121567, 1999.

Chang, T. and Wu, C. C.: Rank-Ordered Multifractal Spectrum for Intermittent Fluctuations, Phys. Rev. E, 77, 045401, https://doi.org/10.1103/PhysRevE.77.045401, 2008.

Chapman, S. C., Hnat, B., and Kiyani, K.: Solar cycle dependence of scaling in solar wind fluctuations, Nonlin. Processes Geophys., 15, 445-455, https://doi.org/10.5194/npg-15-445-2008, 2008.

Conlon, P. A., Gallagher, P. T., McAteer, R. T. J., Ireland, J., Young, C. A., Kestener, P., Hewett, R. J., and Maguire, K.: Multifractal Properties of Evolving Active Regions, Sol. Phys., 248, 297-309, https://doi.org/10.1007/s11207-007-9074-7, 2008.

Dimitropoulou, M., Georgoulis, M., Isliker, H., Vlahos, L., Anastasiadis, A., Strintzi, D., and Moussas, X.: The correlation of fractal structures in the photospheric and the coronal magnetic field, Astron. Astrophys., 505, 1245-1253, https://doi.org/10.1051/0004-6361/200911852, 2009.

Domínguez, M., Muñoz, V., and Valdivia, J. A.: Temporal Evolution of Fractality in the Earth's Magnetosphere and the Solar Photosphere, J. Geophys. Res., 119, 3585-3603, https://doi.org/10.1002/2013JA019433, 2014.

Domínguez, M., Nigro, G., Muñoz, V., and Carbone, V.: Study of Fractal Features of Magnetized Plasma Through an MHD Shell Model, Phys. Plasmas, 24, 072308, https://doi.org/10.1063/1.4993200, 2017.

Domínguez, M., Nigro, G., Muñoz, V., and Carbone, V.: Study of the Fractality of Magnetized Plasma using an MHD Shell Model Driven by Solar Wind Data, Phys. Plasmas, 25, 092302, https://doi.org/10.1063/1.5034129, 2018.

Donner, R. V., Balasis, G., Stolbova, V., Georgiou, M., Wiedermann, M., and Kurths, J.: Recurrence-Based Quantification of Dynamical Complexity in the Earth's Magnetosphere at Geospace Storm Timescales, J. Geophys. Res., 124, 90-108, https://doi.org/10.1029/2018JA025318, 2018.

Echer, E., Alves, M. V., and Gonzalez, W. D.: Geoeffectiveness of Interplanetary Shocks during Solar Minimum (19951996) and Solar Maximum (2000), Sol. Phys., 221, 361-380, https://doi.org/10.1023/B:SOLA.0000035045.65224.f3, 2004.

Eftaxias, K., Contoyiannis, Y., Balasis, G., Karamanos, K., Kopanas, J., Antonopoulos, G., Koulouras, G., and Nomicos, C.: Evidence of fractional-Brownian-motion-type asperity model for earthquake generation in candidate pre-seismic electromagnetic emissions, Nat. Hazards Earth Syst. Sci., 8, 657-669, https://doi.org/10.5194/nhess-8-657-2008, 2008.

Eftaxias, K. A., Kapiris, P. G., Balasis, G. T., Peratzakis, A., Karamanos, K., Kopanas, J., Antonopoulos, G., and Nomicos, K. D.: Unified approach to catastrophic events: from the normal state to geological or biological shock in terms of spectral fractal and nonlinear analysis, Nat. Hazards Earth Syst. Sci., 6, 205-228, https://doi.org/10.5194/nhess-6-205-2006, 2006.

Georgoulis, M. K.: Are Solar Active Regions with Major Flares More Fractal, Multifractal, or Turbulent Than Others?, Sol. Phys., 276, 161-181, https://doi.org/10.1007/s11207-010-97052, 2012.

Gledzer, E. B.: System of Hydrodynamic Type Allowing 2 Quadratic Integrals of Motion, Sov. Phys. Dokl. SSSR, 18, 216217, 1973.

Gonzalez, W. D., Joselyn, J. A., Kamide, Y., Kroehl, H. W., Rostoker, G., Tsurutani, B. T., and Vasyliunas, V. M.: What Is A Geomagnetic Storm?, J. Geophys. Res., 93, 5771-5792, https://doi.org/10.1029/93JA02867, 1994.

Gonzalez, W. D., Dal Lago, A., Clúa de Gonzalez, A. L., Vieira, L. E. A., and Tsurutani, B. T.: Prediction of Peak-Dst from Halo CME/Magnetic Cloud-Speed Observations, J. Atmos. Sol.-Terr. Phy., 66, 161-165, https://doi.org/10.1016/j.jastp.2003.09.006, 2004.

Gündüz, G. and Gündüz, U.: The Mathematical Analysis of the Structure of Some Songs, Physica A, 357, 565-592, https://doi.org/10.1016/j.physa.2005.03.042, 2005.

Hsü, K. J. and Hsü, A. J.: Fractal Geometry of Music, P. Natl. Acad. Sci. USA, 87, 938-941, https://doi.org/10.1073/pnas.87.3.938, 1990.

Huttunen, K. E. J., Koskinen, H. E. J., and Schwenn, R.: Variability of Magnetospheric Storms Driven by Different Solar Wind Perturbations, J. Geophys. Res., 107, 1121, https://doi.org/10.1029/2001JA900171, 2002.

Kane, R. P.: How Good is the Relationship of Solar and Interplanetary Plasma Parameters with Geomagnetic Storms?, J. Geophys. Res., 110, 02213, https://doi.org/10.1029/2004JA010799, 2005.

Kiyani, K., Chapman, S. C., Hnat, B., and Nicol, R. M.: SelfSimilar Signature of the Active Solar Corona within the Inertial Range of Solar-Wind Turbulence, Phys. Rev. Lett., 98, 211101, https://doi.org/10.1103/PhysRevLett.98.211101, 2007.

Kozelov, B. V.: Fractal approach to description of the auroral structure, Ann. Geophys., 21, 201-2023, https://doi.org/10.5194/angeo-21-2011-2003, 2003.

Lepreti, F., Carbone, V., Giuliani, P., Sorriso-Valvo, L., and Veltri, P.: Statistical Properties of Dissipation Bursts within Turbulence: Solar Flares and Geomagnetic Activity, Planet. Space Sci., 52, 957-962, https://doi.org/10.1016/j.pss.2004.03.001, 2004.

Macek, W. M.: Modeling Multifractality of the Solar Wind, Space Sci. Rev., 122, 329-337, https://doi.org/10.1007/s11214-0068185-z, 2006.

Macek, W. M.: Multifractality and intermittency in the solar wind, Nonlin. Processes Geophys., 14, 695-700, https://doi.org/10.5194/npg-14-695-2007, 2007.

Macek, W. M. and Wawrzaszek, A.: Evolution of Asymmetric Multifractal Scaling of Solar Wind Turbulence in the Outer Heliosphere, J. Geophys. Res., 114, 03108, https://doi.org/10.1029/2008JA013795, 2009.

Macek, W. M., Bruno, R., and Consolini, G.: Generalized Dimensions for Fluctuations in the Solar Wind, Phys. Rev. E, 72, 017202, https://doi.org/10.1103/PhysRevE.72.017202, 2005.

Materassi, M. and Consolini, G.: Magnetic Reconnection Rate in Space Plasmas: A Fractal Approach, Phys. Rev. Lett., 99, 175002, https://doi.org/10.1103/PhysRevLett.99.175002, 2007. 
McAteer, R. T. J., Gallagher, P. T., and Ireland, J.: Statistics of Active Region Complexity: A Large-Scale Fractal Dimension Survey, Astrophys. J., 631, 628-635, https://doi.org/10.1086/432412, 2005.

McAteer, R. T. J., Gallagher, P. T., and Conlon, P. A.: Turbulence, Complexity, and Solar Flares, Adv. Space Res., 45, 1067-1074, https://doi.org/10.1016/j.asr.2009.08.026, 2010.

Nanjo, K. and Nagahama, H.: Fractal Properties of Spatial Distributions of Aftershocks and Active Faults, Chaos Soliton. Fract., 19, 387, https://doi.org/10.1016/S0960-0779(03)00051-1, 2004.

Neto, C. R., Guimarães-Filho, Z. O., Caldas, I. L., Nascimento, I. C., and Kuznetsov, Y. K.: Multifractality in Plasma Edge Electrostatic Turbulence, Phys. Plasmas, 15, 082311, https://doi.org/10.1063/1.2973175, 2008.

Nigro, G.: A Shell Model for a Large-Scale Turbulent Dynamo, Geophys. Astro. Fluid, 107, 101-113, https://doi.org/10.1080/03091929.2012.664141, 2013.

Nigro, G. and Carbone, V.: Magnetic Reversals in a Modified Shell Model for Magnetohydrodynamics Turbulence, Phys. Rev. E, 82, 016313, https://doi.org/10.1103/PhysRevE.82.016313, 2010.

Nigro, G. and Carbone, V.: Finite-Time Singularities and Flow Regularization in a Hydromagnetic Shell Model at Extreme Magnetic Prandtl Numbers, New J. Phys., 17, 073038, https://doi.org/10.1088/1367-2630/17/7/073038, 2015.

Nigro, G. and Veltri, P.: A Study of the Dynamo Transition in a Self-Consistent Nonlinear Dynamo Model, Astrophys. J. Lett., 740, L37, https://doi.org/10.1088/2041-8205/740/2/L37, 2011.

Nigro, G., Malara, F., Carbone, V., and Veltri, P.: Nanoflares and MHD Turbulence in Coronal Loops: A Hybrid Shell Model, Phys. Rev. Lett., 92, 194501, https://doi.org/10.1103/PhysRevLett.92.194501, 2004.

Obukhov, A. M.: Some General Properties of Equations Describing The Dynamics of the Atmosphere, Akad. Nauk. SSSR, Izv. Serria Fiz. Atmos. Okeana, 7, 695-704, 1971.

OMNIWeb Plus Data Service: OMNI Data, Goddard Space Flight Center, available at: https://cdaweb.gsfc.nasa.gov/istp_public/, last access: 4 March 2020.

Pastén, D., Muñoz, V., Cisternas, A., Rogan, J., and Valdivia, J. A.: Monofractal and Multifractal Analysis of the Spatial Distribution of Earthquakes in the Central Zone of Chile, Phys. Rev. E, 84, 066123, https://doi.org/10.1103/PhysRevE.84.066123, 2011.

Rangarajan, G. K. and Barreto, L. M.: Long Term Variability in Solar Wind Velocity and IMF Intensity and the Relationship between Solar Wind Parameters \& Geomagnetic Activity, Earth Planets Space, 52, 121, https://doi.org/10.1186/BF03351620, 2000 .
Rathore, B. S., Gupta, D. C., and Parashar, K. K.: Relation Between Solar Wind Parameter and Geomagnetic Storm Condition during Cycle-23, International Journal of Geosciences, 5, 1602-1608, https://doi.org/10.4236/ijg.2014.513131, 2014.

Rathore, B. S., Gupta, D. C., and Kaushik, S. C.: Effect of Solar Wind Plasma Parameters on Space Weather, Res. Astron. Astrophys., 15, 85, https://doi.org/10.1088/1674-4527/15/1/009, 2015.

Sahimi, M., Robertson, M. C., and Sammis, C. G.: Fractal Distribution of Earthquake Hypocenters and its Relation to Fault Patterns and Percolation, Phys. Rev. Lett., 70, 2186-2189, https://doi.org/10.1103/PhysRevLett.70.2186, 1993.

Snyder, C. W., Neugebauer, M., and Rao, U. R.: The Solar Wind Velocity and Its Correlation with Cosmic-Ray Variations and with Solar and Geomagnetic Activity, J. Geophys. Res., 68, 63616370, 1963.

Su, Z.-Y. and Wu, T.: Music Walk, Fractal Geometry in Music, Physica A, 380, 418-428, https://doi.org/10.1016/j.physa.2007.02.079, 2007.

Space Weather Prediction Center (SWPC): Sunspot number data, U.S. Dept. of Commerce, NOAA, available at: ftp://ftp.swpc. noaa.gov/pub/weekly/RecentIndices.txt, last access: 4 March 2020.

Szczepaniak, A. and Macek, W. M.: Asymmetric multifractal model for solar wind intermittent turbulence, Nonlin. Processes Geophys., 15, 615-620, https://doi.org/10.5194/npg-15-615-2008, 2008.

Tsurutani, B. T., Gonzalez, W., Tang, F., Akasofu, S., and Smith, E. J.: Origin of Interplanetary Southward Magnetic Fields Responsible for Major Magnetic Storms near Solar Maximum (1978-1979), J. Geophys. Res., 93, 8519-8531, https://doi.org/10.1029/JA093iA08p08519, 1988.

Uritsky, V. M., Klimas, A. J., and Vassiliadis, D.: Analysis and Prediction of High-Latitude Geomagnetic Disturbances based on a Self-Organized Criticality Framework, Adv. Space Res., 37, 539-546, https://doi.org/10.1016/j.asr.2004.12.059, 2006.

Yamada, M. and Ohkitani, K.: Lyapunov Spectrum of a Model of Two-Dimensional Turbulence, Phys. Rev. Lett., 60, 983-986, https://doi.org/10.1103/PhysRevLett.60.983, 1988.

Yankov, V. V.: Magnetic Field Dissipation and Fractal Model of Current Sheets, Phys. Plasmas, 4, 571, https://doi.org/10.1063/1.872155, 1997.

Zaginaylov, G., Grudiev, A., Shünemann, K., and Turbin, P.: Fractal Properties of Trivelpiece-Gould Waves in Periodic Plasma-Filled Waveguides, Phys. Rev. Lett., 88, 195005, https://doi.org/10.1103/PhysRevLett.88.195005, 2002. 